\title{
Panchromatic imaging and modeling of SSTtau J042021+281349: A new prototypical edge-on protoplanetary disk
}

\author{
G. Duchêne ${ }^{1,2}$, K. Stapelfeldt ${ }^{3}$, A. Isella ${ }^{4}$, M. Perrin ${ }^{5}$, F. Ménard ${ }^{2,6}$, \\ D. Padgett ${ }^{3}$, C. Pinte ${ }^{2,6}$, S. Wolff ${ }^{7}$, J. Krist ${ }^{8}$, A. Ghez ${ }^{9}$ \\ \& Q. Konopacky ${ }^{10}$ \\ ${ }^{1}$ UC Berkeley, ${ }^{2}$ IPAG, ${ }^{3}$ NASA GSFC, ${ }^{4}$ Caltech, ${ }^{5}$ STScI, ${ }^{6}$ U. de Chile, ${ }^{7}$ Johns Hopkins \\ University, ${ }^{8} \mathrm{JPL} /$ Catltech, ${ }^{9} \mathrm{UCLA},{ }^{10}$ Dunlap Institute
}

\begin{abstract}
We present new high-resolution observations and modeling of SSTtau J042021+ 281349, a 400 AU-radius edge-on protoplanetary disk. We have gathered visible and near-infrared scattered light images of the system with the Hubble Space Telescope and Keck adaptive optics system, as well as a $1.3 \mathrm{~mm}$ continuum map with CARMA. Compared to the well-known HH 30 disk, this new system is remarkable because of its spectacular bipolar jet and the high degree of lateral symmetry of the disk. Indeed, we argue that this system is a "cleaner" prototype for edge-on disks. In addition, the apparent achromaticity of dust properties (most notably the almost grey opacity law) from the visible to the near-infrared in this disk suggests that it is in an advanced stage of dust evolution.
\end{abstract}

Keywords. planetary systems: protoplanetary disks; stars: pre-main-sequence

\section{Introduction}

By naturally occulting the bright central star, edge-on protoplanetary disks offer a vantage viewpoint to the structure of these objects. Specifically, the vertical structure of the disk is directly exposed, allowing analysis of the disk scale height and probing vertical settling of the dust. High-resolution images yield strong constraints on the dust scattering and opacity properties. Coupled with millimeter mapping, full spectral energy distribution (SED) and radiative transfer modeling, it is further possible to constrain the global structure of the disk (e.g., Duchêne et al. 2010).

We have conducted an HST/ACS imaging survey of Spitzer-selected new candidate edge-on protoplanetary disks to increase the number of such known systems (see contributions by K. Stapelfeldt and S. Wolff in this volume). One of the targets was SSTtau J042021+281349 (hereafter Tau042021), an M0-M1 member of the Taurus star-forming region characterized by a rich emission line spectrum, a very low apparent stellar luminosity and a massive infrared excess $\left(L_{\star}=0.004 L_{\odot}, L_{I R} / L_{b o l}=0.44\right.$; Rebull et al. 2010). These latter two points are characteristics of edge-on disk systems and, indeed, Tau042021 was first resolved as such in ground-based images (Luhman et al. 2009).

\section{Observational results and initial modeling results}

We have obtained $\leqslant 0.1$ "-resolution visible and near-infrared images of Tau042021 with HST and Keck adaptive optics, as well as an 0.4 "-resolution $1.3 \mathrm{~mm}$ map of the system with CARMA (see left panel in Fig. 1). The HST images show a highly symmetric, 400 AU-radius, edge-on disk as well as a collimated Herbig-Haro jet extending up to 8" from the central star with multiple knots along both the jet and counter-jet. In addition to 

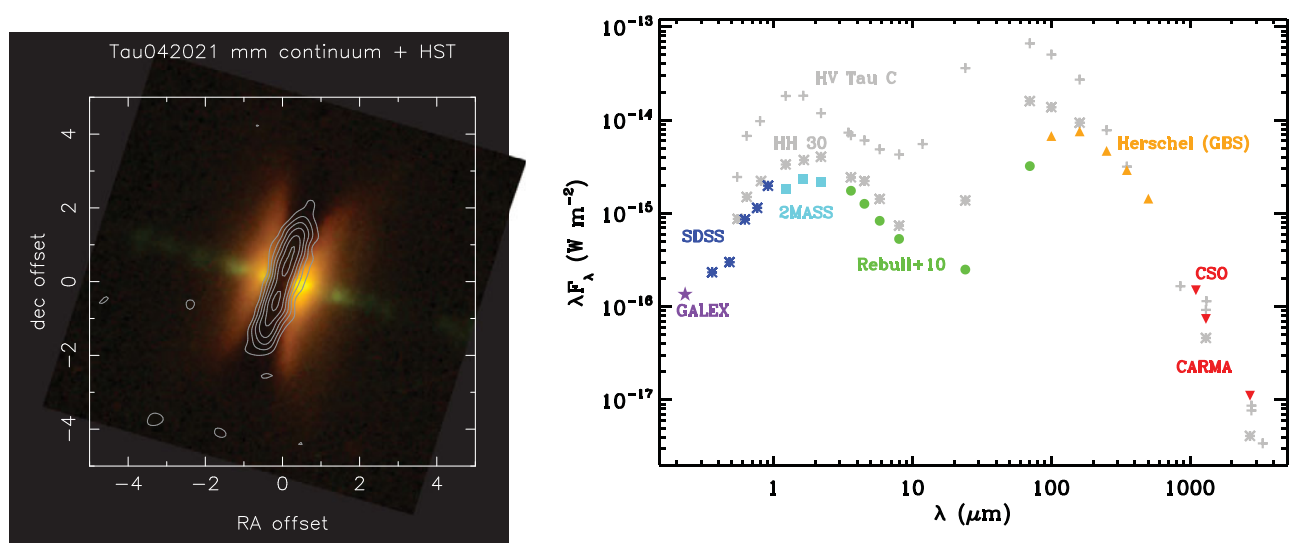

Figure 1. Left: Two-color HST/ACS image (F606W: green; F814W: red) and $1.3 \mathrm{~mm}$ continuum contour map of Tau042021. Right: Complete SED for Tau042021 (color symbols) compared to those of the HH 30 and HV Tau C edge-on protoplanetary disks (gray asterisks and plus signs).

the typical two parallel nebulae separated by the darklane that defines the disk midplane, our HST images reveal a flat-topped, low surface brightness and markedly blue-colored, nebulosity high up above the disk. The high-resolution millimeter map shows a very flat structure (unresolved along the vertical axis of the disk) with an intriguing double-peak structure. Finally, the SED of Tau042021, with our new CSO and CARMA data, shows a shallow submillimeter spectral slope $\left(\alpha_{\mathrm{mm}} \approx 1.9\right.$, see right panel of Fig. 1$)$.

The system's scattered light images reveal a remarkable lack of chromaticity in the dust scattering and opacity properties (e.g., the dark lane width shrinks by only $20 \%$ between 0.8 and $2.2 \mu \mathrm{m}$ ), suggesting that the dust population in the disk has substantially evolved. The weak nebulosity seen above the disk could be an extended disk "atmosphere", for instance related to photoevaporation, or evidence that the disk extends further out radially than suggested by the images, in which case a tapered surface density profile would be required to match all observations (e.g., Isella et al. 2009). Extensive simultaneous modeling of the SED and scattered light images is ongoing and will help distinguish between these two possibilities. Preliminary results indicate a "normal" stellar luminosity $\left(L_{\star} \approx 0.5 L_{\odot}\right)$, a massive $\operatorname{disk}\left(M_{\mathrm{dust}} \approx 310^{-4} M_{\odot}\right)$ and very little foreground extinction $\left(A_{V} \leqslant 1 \mathrm{mag}\right)$, in line with the ultraviolet detection of the system with GALEX. Finally, the double-peak structure of the CARMA $1.3 \mathrm{~mm}$ map is reminiscent of other edge-on protoplanetary disks and this ubiquity suggests that it is more likely an optical depth effect rather than evidence for a central binary source (see also Gräfe et al. 2013).

Compared to $\mathrm{HH} 30$, the disk surrounding Tau042021 is larger, observed closer to edgeon $\left(i \approx 88^{\circ}\right.$ vs. $\left.\approx 80^{\circ}\right)$ and does not show pronounced lateral asymmetry. We thus argue that Tau042021 is a better prototype of edge-on protoplanetary disks than HH 30 and anticipate that it will become a new benchmark to compare other similar systems.

\section{References}

Duchêne, G., McCabe, C., Pinte, C., et al. 2010, ApJ, 712, 112

Gräfe, C., Wolf, S., Guilloteau, S., et al. 2013, A\&广A, 553, A69

Isella, A., Carpenter, J., \& Sargent, 2009, ApJ, 701, 260

Luhman, K., Mamajek, E., Allen, P., \& Cruz, K., 2009, ApJ, 703, 399

Rebull, L., Padgett, D., McCabe, C., et al. 2010, ApJS, 186, 259 